\title{
DIE RECHTLICHE ZULÄSSIGKEIT DES SOG. ALTERNATIVVERMÄCHTNISSES IM POLNISCHEN ERBRECHT VOR DEM HINTERGRUND DES DEUTSCHEN RECHTS
}

\begin{abstract}
Zusammenfassung
In letzter Zeit haben fast alle populären juristischen Medien die Entscheidung des (polnischen) Obersten Gerichtshofes kommentiert, der das „Alternativvermächtnis" zugelassen hat Bei dem Alternativvermächtnis handelt sich um ein Vermächtnis, bei dem der Erblasser anderen Personen (Vermächtnisnehmern, Erben, sogar dritten Personen) die Wahl zwischen alternativ angegebenen Vermächtnisgegenständen überlässt.

In der polnischen Lehre wird eine solche Rechtsfigur richtigerweise als unzulässig angesehen. Der Oberste Gerichtshof dagegen hat geurteilt, dass "ein einfaches Vermächtnis (Artikel $968 \S 1$ k.c.), in welchem der Leistungsgegenstand alternativ beschrieben wird, wirksam sei. Diese Entscheidung verdient nach meiner Auffassung ebenso wie ihre Begründung keine Billigung. Interessanterweise wurde sie sofort in den populären Medien mit Zufriedenheit und ausdrücklicher Zustimmung zur Kenntnis genommen. Es lohnt daher, sich über die theoretische Konstruktion des Alternativvermächtnisses im Lichte der Grundstrukturen des polnischen Erbrechts Gedanken zu machen und sie mit Lösungen zu konfrontieren, die sich im deutschen Recht finden. Letzteres lässt expressis verbis unter der Bezeichnung Wahlvermächtnis das Alternativvermächtnis $z u$, obwohl es im allgemeinen wie auch in vielen Einzelfragen dem polnischen sehr nahe ist.
\end{abstract}

Keywords

Erbrecht - polnisches Erbrecht - deutsches Erbrecht - Alternativvermächtnis - Wahlvermächtnis

* Professor Dr. habil. Tomasz Justyński (LL.M.), Nicolaus-Copernicus-Universität Toruń, Dekan der Fakultät für Jura und Verwaltung. 


\section{Allgemeine Vorbemerkungen}

Probleme des Erbrechts erleben in den letzten Jahren in Polen eine wahre Renaissance. Nicht zu erkennen, dass in den fünfundzwanzig Jahren nach den axiologischen Veränderungen im polnischen Recht die gesellschaftlich-wirtschaftliche Bedeutung des Erbrechts ganz erheblich gestiegen ist, wäre in der Tat schwierig. Überwintert in all den Jahren des realen Sozialismus, stellt es sich gegenwärtig als ein wichtiges und immer stärker geschätztes Instrument dar, ein Erbe im Todesfall sicherzustellen. Viele bedeutsame und vielfach auch juristisch komplizierte Fragen werden unter Beteiligung breiter gesellschaftlicher Kreise in den Spalten der Medien diskutiert. So war es beispielsweise mit der gesetzlichen Regelung der Erbfolge, insbesondere mit dem Kreis der gesetzlichen Erben. So geschah es auch mit der sog. Schenkung auf den Todesfall (donatio mortis causa) und mit der Beschränkung der Erbenhaftung auf den Nachlass. In letzter Zeit haben fast alle populären juristischen Medien die Entscheidung des (polnischen) Obersten Gerichtshofes kommentiert, der das "Alternativvermächtnis" zugelassen hat ${ }^{1}$.

Bei dem Alternativvermächtnis handelt sich um ein Vermächtnis, bei dem der Erblasser anderen Personen (Vermächtnisnehmern, Erben, sogar dritten Personen) die Wahl zwischen alternativ angegebenen Vermächtnisgegenständen überlässt.

In der polnischen Lehre wird eine solche Rechtsfigur richtigerweise als unzulässig angesehen. Der Oberste Gerichtshof dagegen hat geurteilt, dass „ein einfaches Vermächtnis (Artikel 968 \& 1 k.c. ${ }^{2}$, in welchem der Leistungsgegenstand alternativ beschrieben wird, wirksam sei". Diese Entscheidung verdient nach meiner Auffassung ebenso wie ihre Begründung keine Billigung. Interessanterweise wurde sie sofort

\footnotetext{
1 Beschluss vom 16.10.2014, III CZP 70/14, Orzecznictwo Sądów Polskich [Die Rechtsprechung Polnischer Gerichte/Entscheidungssammlung] 2015, Heft 4 mit Anmerkung T. Justyński. Vergleiche auch: T. Justyński, Uwagi w sprawie prawnej dopuszczalności zawierania umów darowizny ze skutkiem mortis causa [Anmerkungen zur Frage der rechtlichen Zulässigkeit des Abschlusses von Schenkungsverträgen auf den Todesfall], Przegląd Sądowy [Gerichtsumschau] 2015, Heft 4, S. 7-16.

2 Kodeks cywilny [Polnisches Zivilgesetzbuch] zitiert als k.c.
} 
in den populären Medien mit Zufriedenheit und ausdrücklicher Zustimmung zur Kenntnis genommen ${ }^{3}$. Es lohnt daher, sich über die theoretische Konstruktion des Alternativvermächtnisses im Lichte der Grundstrukturen des polnischen Erbrechts Gedanken zu machen und sie mit Lösungen $\mathrm{zu}$ konfrontieren, die sich im deutschen Recht finden. Letzteres lässt expressis verbis unter der Bezeichnung Wahlvermächtnis das Alternativvermächtnis zu, obwohl es im allgemeinen wie auch in vielen Einzelfragen dem polnischen sehr nahe ist.

\section{Der BEGRIFF DES ALternativVERMÄCHTNISSES}

Die polnische Gesetzessprache kennt den Begriff "Alternativvermächtnis“ (optio legata, alternate legacy) nicht. Das ist nicht verwunderlich, denn im polnischen Erbrecht gibt es diese besondere Art eines Vermächtnisses nicht. In der polnischen Lehre (ähnlich wie in der internationalen) besteht trotzdem kein Streit darüber, wie der Ausdruck zu verstehen ist. Ein Alternativvermächtnis liegt vor, wenn der Erblasser den Gegenstand eines Vermächtnisses nicht genau bezeichnet, sondern anderen Personen (Vermächtnisnehmern, Erben, sogar dritten Personen) die Wahl zwischen alternativ angegebenen Gegenständen überlässt. Das Wesen des Alternativvermächtnisses ist es daher, dass die endgültige Bestimmung der Leistung dem freien Ermessen eines oder mehrerer Dritter überlassen bleibt. Der Wille dieser dritten Personen „ergänzt“ sozusagen den Willen des Erblassers, womit dieser natürlich vom Grundsatz her einverstanden ist.

Von dem so verstandenen Alternativvermächtnis, mit dem sich, wie gesagt, der polnische Oberste Gerichtshof befasst hat ${ }^{4}$, ist eine nur prima facie ähnliche Verfügung von Todes wegen $\mathrm{zu}$ unterscheiden.

\footnotetext{
3 Statt vieler s. z.B. M. Domagalski, Zapiszesz pieniadze lub dom - do wyboru [Du vermachst Geld oder ein Haus - nach Wahl], http://archiwum.rp.pl/artykul/1257645-Zapiszeszpienadze-lub-dom-\%E2\%80\%93-do-wyboru.html [letzter Abruf: 21.10.2015].

4 In dem zugrunde liegenden Fall hatte ein Testator einen Sohn zum Gesamterben eingesetzt und ihn mit einem Vermächtnis zugunsten einer Tochter beschwert. Gegenstand des Vermächtnisses sollte eine Bau-Immobilie sein oder ihr Gegenwert, wohl nach Wahl des oder der Erben oder der Erbin (wobei weder klar war, wer zur Wahl berechtigt war, noch ob es sich um den Wert derselben oder irgend einer anderen, dem Verstorbenen gehörenden Immobilie handelte).
} 
Auch hier gestaltet der Erblasser das Vermächtnis in einem gewissen Sinne "alternativ“. Für den Fall, dass ihm zum Zeitpunkt seines Todes bestimmte Gegenstände nicht mehr gehören, kann er einen angemessenen „Ersatz" benennen und damit eine andere Vermögensleistung zum Vermächtnisgegenstand machen. Eine solche Verfügung würde ohne weiteres den in Artikel $968 \S 1$ k.c. ${ }^{5}$ formulierten Anforderungen genügen.

Diese Art der Verfügung ruft eine deutliche Assoziation mit der Rechtsfigur des sog. Ersatzerben hervor. Sein Wesen ist bekanntlich die Bestimmung eines „Reserve-Erben“ für den Fall, dass der eigentlich bedachte Erbe nicht erben will oder kann. Eine solche Bestimmung wird expressis verbis vom polnischen Gesetzgeber akzeptiert (Artikel 963 k.c. ${ }^{6}$ ). Daraus wird geschlossen, dass, wenn es rechtlich möglich ist, sogar einen „Reserve“-Erben zu bestellen, es erst recht zulässig sein muss, lediglich einen „Reserve-Vermächtnisgegenstand“ zu benennen. Das Fehlen einer ausdrücklichen Vorschrift für diesen Fall dürfe nicht so verstanden werden, als solle ein mögliches „Vermächtnis mit einem ReserveGegenstand" ausgeschlossen werden. Es genügt an dieser Stelle der Hinweis, dass eine ausdrückliche gesetzliche Regelung der Ersatzerbschaft nicht schon deswegen nötig wäre, weil ohne sie die Rechtsfigur nicht zulässig wäre. Das Problem liegt vielmehr darin, sie von der im polnischen Erbrecht verbotenen treuhänderischen Ersatzerbschaft zu unterscheiden.

Möglicherweise hätte man in dem im polnischen Erbrecht geltenden Verbot einer an Bedingungen gebundenen Einsetzung eines Erben (Artikel 962 k.c. ${ }^{7}$ ) einen weiteren Grund für die Notwendigkeit einer ausdrücklichen Regelung der Ersatzerbschaft finden können. Man kann die Sache nämlich so sehen, dass der Ersatzerbe an sein Erbteil unter der „Bedingung“ gelangt, dass der „ursprüngliche“ Erbe nicht erben wird.

\footnotetext{
5 Artikel 968 \& 1 k.c.: Der Erblasser kann durch testamentarische Verfügung den gesetzlichen oder testamentarischen Erben verpflichten, eine bestimmte Leistung zugunsten einer benannten Person zu erbringen (einfaches Vermächtnis).

6 Artikel 963 k.c.: Es kann ein testamentarischer Erbe eingesetzt werden für den Fall, dass eine andere als gesetzlicher oder testamentarischer Erbe eingesetzte Person kein Erbe sein will oder kann (Ersetzung).

7 Artikel 962 Satz 1 k.c.: Ein bei der Einsetzung eines testamentarischen Erben gemachter Bedingungs- oder Termin-Vorbehalt wird als nicht existent angesehen.
} 
Bei einer solchen Sicht wäre eine lex specialis für die Durchbrechung dieses Verbotes notwendig.

Das Verbot, einen Erben unter Bedingungen einzusetzen, hindert offensichtlich nicht die Anerkennung eines "Vermächtnisses mit Reservegegenstand“8. Es besteht auch kein Zweifel daran, dass es, um ein "Vermächtnis mit Reservegegenstand" als zulässig anzuerkennen, keiner ausdrücklichen Rechtsnorm bedarf, die expressis verbis diese Konstruktion vorsieht. Hier wird nämlich nicht die Befugnis an andere Personen übertragen, den Willen des Erblassers zu ergänzen. Damit wird nicht gegen den Grundsatz verstoßen, dass ein Testament persönlich abgefasst werden muss (s. u.).

Ein bei Abfassung "alternatives Vermächtnis" kann in Folge einer Veränderung der Umstände im Zeitpunkt des Erbfalls "alternativlos" geworden sein. Eine Auswahl (gleichgültig, wem sie überlassen wurde) wäre dann gegenstandslos. Auch hier wäre anzuerkennen, dass das Vermächtnis wirksam ist.

\section{DiE KOLLISION ERB- UND SCHULDRECHTLICHER REGELN BEI DER GESTALTUNG UND AUSLEGUNG MORTIS CAUSA VORGENOMMENER HANDLUNGEN}

In letzter Zeit hat es sowohl in einigen Entscheidungen des polnischen Obersten Gerichtshofes als auch in einzelnen Äußerungen im polnischen erbrechtlichen Schrifttum Versuche gegeben, eine "Liberalisierung“" des Erbrechts vorzunehmen, indem man erbrechtliche Institute aus dem Blickwinkel des Schuldrechts interpretiert. So verfuhr beispielsweise der Oberste Gerichtshof bei seiner Begründung für die Zulässigkeit des Instituts der Schenkung mortis causa10. Das Gericht behandelte diese eindeutig erbrechtliche Rechtsfigur sozusagen als Abart

\footnotetext{
8 Denn das Vermächtnis selbst kann unter einer Bedingung (oder mit einem Terminvorbehalt) errichtet werden (s. Artikel 975 k.c.).

9 Den wenig glücklichen Ausdruck "Liberalisierung“ benutzt der Oberste Gerichtshof selbst in seiner in Fußnote 1 zitierten Entscheidung.

10 Vgl. die Entscheidung des Obersten Gerichtshofes vom 13.12.2013, III CZP 79/13, Orzecznictwo Sądów Polskich [Die Rechtsprechung Polnischer Gerichte Entscheidungssammlung] 2014, Heft 10, Pos. 91 mit ablehnender Anmerkung T. Justyński.
} 
der (schuldrechtlichen) Schenkung inter vivos. Das ermöglicht es ihm, den ganzen einschlägigen konzeptionellen Apparat für Handlungen unter Lebenden anzuwenden und in der Konsequenz, schon de lege lata die Zulässigkeit einer Schenkung mortis causa zu begründen ${ }^{11}$.

Ähnlich verfuhr der polnische Oberste Gerichtshof mit dem Alternativvermächtnis, indem er außerhalb des Erbrechts nach den Regeln des Schuldrechts die Begründung dafür suchte, dass ein Erblasser einem Vermächtnisnehmer die Wahl unter zwei oder mehr Leistungsgegenständen überlassen kann ${ }^{12}$.

Das legt es nahe, das Verhältnis der Regelungen des Erbrechts und des Schuldrechts zueinander im Hinblick auf das Alternativvermächtnis näher zu betrachten.

Die Feststellung, dass das Erbrecht kein isolierter Bestandteil des Zivilrechts ist, ist eine Selbstverständlichkeit. Trotz seiner immer wieder zu Recht betonten Eigenart (es geht um mortis causa wirksame Handlungen) stellt es kein selbständiges, in sich abgeschlossenes Regelwerk dar. Eine „Unterstützung“ der erbrechtlichen Regelungen durch Regeln aus anderen Teilen des Zivilrechts (insbesondere aus dem Allgemeinen Teil, aber auch aus dem Schuldrecht) ist oft notwendig und kann im Prinzip nicht bestritten werden. Es ist jedoch schwer, sich des Eindrucks zu erwehren, dass der polnische Oberste Gerichtshof bei der Auslegung der Vorschriften über das Vermächtnis mit allzu großer Leichtigkeit zwischen dem Erbrecht und dem Schuldrecht hin und her wandert. Wie es scheint, liegt hier auch der Grund für seine Folgerung, das Alternativvermächtnis zuzulassen.

Aus der Beobachtung, dass der (lebende) Erblasser dem (ebenfalls lebenden) Vermächtnisnehmer eine bestimmte Vermögensleistung verspricht, zieht der Oberste Gerichtshof (im Beschluss vom 16. Oktober 201413) den Schluss, dass auch die G e s ta $1 \mathrm{t} \mathrm{u} \mathrm{n} \mathrm{g} \mathrm{des}$ Vermächtnisses den Regeln des Schuldrechts unterliege. Er fügt

\footnotetext{
11 Ausführlicher zu dem Thema: T. Justyński, Uwagi w sprawie prawnej dopuszczalności zawierania umów darowizny $z$ skutkiem mortis causa [Bemerkungen zur rechtlichen Zulässigkeit des Abschlusses von Schenkungsverträgen von Todes wegen], Przegląd Sądowy [Gerichtsumschau] 2015, Heft 4, S. 7 ff.

12 Der in Fußnote 1 zitierte Beschluss.

13 Beschluss vom 16.10.2014, III CZP 70/14, Fußnote 1.
} 
zwar hinzu, dass dabei die abweichenden Regelungen des Erbrechts $\mathrm{zu}$ berücksichtigen seien, aber offensichtlich fehlte es genau daran. $\mathrm{Da}$ nach dem Schuldrecht alternative Leistungen vereinbart werden können und auch die Überlassung der Leistungsbestimmung an dritte Personen möglich ist, muss es nach Meinung des Gerichtshofes auch im Hinblick auf das Vermächtnis so sein. Dem ist aber nicht so. Die Anforderungen (sowohl in formaler als auch in materieller Hinsicht) an die Gestaltung von mortis causa vorgenommenen Rechtshandlungen bestimmt das Erbrecht; seine Grundsätze müssen bei der Gestaltung berücksichtigt werden. Das Erbrecht wird aber (aus offensichtlichen und somit an dieser Stelle keiner speziellen Beweisführung bedürfenden Gründen) von anderen Grundsätzen beherrscht als das Schuldrecht.

\section{DER GRUNDSATZ DER PERSÖNLICHEN ERSTELLUNG EINES TESTAMENTS UND EINES VERMÄCHTNISSES}

Was das Alternativvermächtnis anbetrifft, so darf man nicht den das polnische Erbrecht beherrschenden Grundsatz des p e r s ö n 1 i c h e n Charakters eines Testaments (Artikel $944 \S 2$ k.c. ${ }^{14}$ ) vergessen. Aus diesem Grundsatz folgt, dass ein Testament (sowohl in seiner Ganzheit als auch in Bezug auf einzelne seiner Bestimmungen) persönlich durch den Testator errichtet werden und seinem persönlichen Willen entsprechen muss. Genau hier liegt einer der fundamentalen Unterschiede zwischen Rechtshandlungen inter vivos und mortis causa.

Für Rechtshandlungen inter vivos gilt der Grundsatz des persönlichen Charakters nicht. Bei einer Handlung inter vivos ist es eine Selbstverständlichkeit, dass der Vollmachtgeber nicht selbst alle Bestandteile ihres Inhaltes bestimmen muss. Das können grundsätzlich auch andere Personen tun. Das zeigt sich schon daran, dass - jedenfalls im Grundsatz - jede Rechtshandlung durch einen Vertreter vorgenommen werden kann, der eine eigene Willenserklärung abgibt und damit de facto den Inhalt der Erklärung gestaltet, auch wenn dem manchmal sehr enge Grenzen gezogen sind.

14 Artikel $944 \S 2$ k.c.: Ein Testament kann nicht durch einen Vertreter aufgesetzt oder widderrufen werden. 
Anders ist es im Erbrecht. Hier sind die Regeln umgekehrt. Grundsatz ist der streng persönliche Charakter einer Rechtshandlung (Artikel $944 \S \S 1$ und 2 k.c.) ${ }^{15}$, Ausnahmen bedürfen einer ausdrücklichen gesetzgeberischen Ermächtigung. Es unterliegt keinem Zweifel, dass der Grundsatz des persönlichen Charakters der Testamentserrichtung auch für das Vermächtnis gilt. Es genügt, daran zu erinnern, dass ein Vermächtnis ausschließlich in einem Testament errichtet werden kann: In den Worten des Artikel 968 \& 1 k.c. „Der Erblasser kann durch testamentarische Verfügung den Erben verpflichten“. Das Legat stellt einen integralen Bestandteil des Testaments dar ${ }^{16}$.

Ebenso offensichtlich ist es, dass der Grundsatz des persönlichen Charakters sowohl für das Testament als Ganzes als auch für seine einzelnen Bestandteile gilt. Und da es so ist, kann niemanden die Schlussfolgerung verwundern, dass auch das Vermächtnis dem in Artikel 944 \& 2 k.c. ausgesprochenen Grundsatz unterworfen ist. Dieser Grundsatz wird bei Zulassung des sog. Alternativvermächtnisses verletzt. Überlässt ein Erblasser die Auswahl eines Leistungsgegenstades dritten Personen, so gestaltet er das Vermächtnis nicht selbst und errichtet folglich das Testament nicht persönlich.

Schon das erlaubt es, eine abschließende Schlussfolgerung zum Problem der Zulässigkeit eines Alternativvermächtnisses de lege lata im polnischen Erbrecht zu ziehen: Im Hinblick auf die fundamentale Bedeutung des Grundsatzes des persönlichen Charakters des Testaments und damit auch eines Vermächtnisses erfordert die Einführung eines Wahlvermächtnisses ein Einschreiten des Gesetzgebers.

Der durch die Judikatur unternommene Versuch einer „Legalisierung“ des Alternativvermächtnisses muss nicht nur als fehlerhaft, sondern auch als gefährlich bezeichnet werden. Denn sie kann zur Erosion des Grundsatzes des persönlichen Charakters von Rechtshandlungen auch

\footnotetext{
15 Zwar bezieht sich die Vorschrift einfach nur auf das Testament, aber angesichts des im polnischen Recht geltenden Grundsatzes des numerus clausus für Handlungen mortis causa (Artikel 941 k.c.) hat sie de facto allgemeine Bedeutung.

16 Statt vieler s. S. Wócik, F. Zoll, [in:] Z. Radwański (Red.), System Prawa Prywatnego. Prawo spadkowe [System des Privatrechts. Erbrecht], Bd. 10, Warszawa: C.H. Beck 2009, S. 374, Anm. 14.
} 
im übrigen Bereich ihrer Geltung führen, insbesondere bei der Erbeinsetzung.

An dieser Stelle könnte man eigentlich die Überlegungen abschließen. Im Beschluss vom 16. Oktober 2014 hat der Oberste Gerichtshof jedoch noch zwei Argumente bemüht, auf die man nicht nicht reagieren kann. Sie haben zwar nur Hilfscharakter, können aber Verwirrung hervorrufen.

\section{DeR WiLle DES VerstorbenEN UND DIE NOTWENDIGE BEGRENZUNG DER TESTIERFREIHEIT}

Der Gerichtshof nennt als Argumente für die Zulässigkeit eines Alternativvermächtnisses de lege lata den Grundsatz einer möglichst vollständigen Verwirklichung des Willens des Verstorbenen und den Grundsatz einer Auslegung in favorem testamenti. Es ist zwar schwerlich $\mathrm{zu}$ bestreiten, dass beide $\mathrm{zu}$ den fundamentalen Regeln des Erbrechts gehören, trotzdem können die Argumente nicht überzeugen, geschweige denn als ausreichend anerkannt werden.

Es ist eine Binsenweisheit, dass im polnischen Erbrecht (ebenso wie in ausländischen Rechtsordnungen) der Wille des Verstorbenen zwar sehr wichtig ist, dass er aber keinen absoluten Grundsatz darstellt, mit dem nicht andere gleichgewichtige Prinzipien konkurrieren könnten. So hat der Gesetzgeber dem Willen des Erblassers vielerlei Grenzen gesetzt, die die Testierfreiheit nicht unerheblich einschränken. Als Beispiel sei nur der numerus clausus der Rechtshandlungen mortis causa genannt (Artikel 941 k.c.). Der Erblasser kann seinen Willen ausschließlich (!) mit Hilfe von Rechtskonstruktionen verwirklichen, welche expressis verbis vom Gesetzgeber vorgegeben sind. Auch ein zweifelsfrei zum Ausdruck gekommener Wille des Testators kann nicht berücksichtigt werden, wenn er sich nicht in dem vom Gesetzgeber vorgegebenen Rahmen hält.

Ebenso bedeutet das Prinzip der persönlichen Errichtung des Testaments eine Beschränkung des Willens des Erblassers. Prima facie klingt diese Feststellung etwas überraschend. Aber sie hört schnell auf, es $\mathrm{zu}$ sein, wenn man bedenkt, dass es doch in concreto der Wille des Testators sein könnte, dass ein anderer sein Testament aufsetzt (und das sowohl - was weniger strittig ist - in rein technischer als auch in inhaltlicher Hinsicht, dass er es also de facto nach seinem Wissen 
und Willen für den Testator gestaltet). Offensichtlich ist das jedoch nicht erlaubt. Entgegen dem Anschein garantiert der Grundsatz des persönlichen Charakters eines Testaments nicht nur die Übereinstimmung mit dem tatsächlichen Willen des Testators, sondern er gibt auch, was nicht weniger wichtig ist, durch die Beschränkung des freien Willens ein Minimum an Sicherheit, dass das Testament genau vom verstorbenen Erblasser stammt. Dieser zweite Aspekt des Grundsatzes des persönlichen Charakters eines Testaments ist leicht zu erkennen, wenn man ihn mit den Regeln für die Form des Testaments in Verbindung setzt: mit dem Erfordernis der Eigenhändigkeit bei der Errichtung eines holographischen Testaments, der Anwesenheit eines Notars bei einem notariellen Testament oder von Zeugen in anderen Fällen.

Den Grundsatz der persönlichen Ausfertigung darf man auch bei der Ausgestaltung des Vermächtnisses nicht außer Betracht lassen. Vor allem darf man diesen Grundsatz nicht dadurch in Frage stellen, dass man sich, wie das der Oberste Gerichtshof im Beschluss vom 16. Oktober 2014 getan hat, auf eine vollständigere Verwirklichung des Willens des Erblassers beruft. Der Gesetzgeber hat die Durchsetzung dieses Willens bis zu einem gewissen Grad begrenzt und sogar geopfert. Das Erfordernis einer persönlichen Testamentserrichtung führt in concreto vielleicht $\mathrm{zu}$ einer evidenten Divergenz zum tatsächlichen Willen des Erblassers. Es sichert aber (oder ermöglicht geradezu) die Beachtung dieses Willens als generelle Regel ${ }^{17}$.

\section{DiE SACHENRECHTLICHE NATUR DES VindikATIONSLEGATS UND DAS SCHULDRECHTLICHE WESEN EINES „EINFACHEN VERMÄCHTNISSES"}

Eines der Argumente, die es dem polnischen Obersten Gerichtshof erlauben, die These von der Zulässigkeit des Alternativvermächtnisses aufzustellen, ist die Behauptung, das einfache Vermächtnis (legatum per damnationem) sei eine "liberalere“ Konstruktion als das kürzlich

17 In diesem Sinne ist Artikel 944 k.c. eine lex specialis zu den Auslegungsregeln in Artikel 948 k.c. 
in das polnische Erbrecht eingeführte Vindikationslegat (legatum per vindicationem). Anders als beim "einfachen Vermächtnis" hat der Gerichtshof bei diesem Vermächtnis keinen Zweifel, dass es nicht als Alternativvermächtnis formuliert werden darf.

Der Oberste Gerichtshof bemerkt zwar und unterstreicht ausdrücklich, dass ein Vindikationslegat nicht alternativ gefasst werden darf, weil seine Wirkungen schon im Augenblick des Todes des Erblassers (Testators) eintreten. Daraus, dass bei einem Vindikationslegat eine Wahl"Alternative“" nicht zulässig ist, zieht er aber den Schluss, dass es bei einem einfachen Vermächtnis entgegengesetzt sein soll. Zur Begründung führt er, wie erwähnt, die "größere Liberalität" dieser Art von Vermächtnis an.

Folgt man dem, könnte man den Eindruck gewinnen, dass es das Ziel des Gesetzgebers bei der Einführung des Vindikationslegats gewesen wäre, dieses Institut strengeren Regeln $\mathrm{zu}$ unterwerfen. Aber dem Gesetzgeber ging es nicht darum. Er wollte vielmehr die Verfügung mortis causa über einen $\mathrm{k}$ o $\mathrm{n} \mathrm{k}$ e $\mathrm{te} \mathrm{n}$ Gegenstand mit $\mathrm{s}$ a c he $\mathrm{n} \mathrm{e} \mathrm{cht} \mathrm{i} \mathrm{ch}$ e $\mathrm{r}$ W $\mathrm{rku} \mathrm{g}$ möglich machen, d. h. er wollte hinsichtlich des vermachten Gegenstandes dieselbe Wirkung erzielen wie bei einer (partiellen) Erbeinsetzung ${ }^{18}$.

Daraus, dass bei dem Vindikationsvermächtnis eine alternative Fassung des Vermächtnisgegenstandes nicht möglich ist, lassen sich infolgedessen keine Rückschlüsse auf die Natur des „einfachen Vermächtnisses“ ziehen, zumindest keine Schlüsse auf dessen Zulässigkeit (oder Nichtzulässigkeit). Ganz abgesehen von der bekannten Schwäche einer Argumentation a contrario fehlen hier die Grundlagen für die Anwendung dieser Schlussfolgerungsmethode. Bei den beiden „Vermächtnissen“ handelt es sich um ganz unterschiedliche Institute, die sich trotz ähnlicher Bezeichnung grundsätzlich voneinander unterscheiden.

18 Zur Natur des Vindikationsvermächtnisses vgl. z.B. E. Skowrońska-Bocian, Prawo spadkowe [Erbrecht], Warszawa: C.H. Beck 2014, S. 120, Rz. 188. 


\section{GESELLSCHAFTLICHER UND WIRTSCHAFTLICHER BEDARF AN EINER NEUEN RECHTSFIGUR IM ERBRECHT}

Schließlich fragt es sich, ob in der polnischen Gesellschaft tatsächlich ein Bedarf an einem „Wahlvermächtnis“ besteht. Das Vindikationslegat19 verdankt seine Einführung dem Bedarf an einer Möglichkeit, mit sachenrechtlicher Wirkung über einen bestimmten, zum Erbe gehörenden Gegenstand zu verfügen, was die Einbeziehung in das Erbe bekanntlich nicht leistet; die Schenkung mortis causa gibt einerseits dem Erblasser die Möglichkeit, eine Pflege oder die Mitarbeit in einem Unternehmen erst im Todesfall zu entlohnen, andererseits dem Bedachten schon zu Lebzeiten die Sicherheit eines unwiderruflichen Rechtsaktes, die ein Testament nicht bietet ${ }^{20}$. Einen Bedarf gibt es auch für eine Reihe anderer Instrumente des Erbrechts, über die in der Öffentlichkeit weniger diskutiert wird, etwa für den "treuhänderischen Ersatzerben“, der bei der Herausbildung des realen Sozialismus - wie bekannt, aus ideologischen Gründen - aus dem polnischen Recht entfernt wurde.

An einem Bedarf für ein Alternativvermächtnis dagegen kann man Zweifel haben. Es gibt sogar triftige Gründe, die gegen seine Zulassung sprechen. Es ist beispielsweise schwer $\mathrm{zu}$ übersehen, dass ein Alternativvermächtnis aus der Natur der Sache zu größerer Rechtsunsicherheit führt als ein „einfaches Vermächtnis“. Bei einem einfachen Vermächtnis beschränkt sich die Unsicherheit darauf, ob der Vermächtnisnehmer fordert, eine Leistung $\mathrm{zu}$ erbringen. Beim Alternativvermächtnis müssten zwei oder evtl. sogar mehr Leistungsgegenstände „reserviert" werden. Der Erbe würde über diese Gegenstände unter Umständen über längere Zeit nicht verfügen können; er wäre praktisch auf die Rolle eines Treuhänders beschränkt. Anders

19 Gesetz über die Änderung des Zivilgesetzbuches sowie einiger anderer Gesetze
vom 18.03.2011, Dziennik Ustaw [Gesetzblatt] Nr. 8, Pos. 458 .
20 Zu dem Gesetzentwurf über die Einfügung der Schenkung mortis causa in das Erbrecht
z.B. T. Justyński, Opinia II z 14 grudnia 2010 r. dotyczaca projektu ustawy zawartego w druku 2116
(senacki projekt ustawy o zmianie ustawy - Kodeks cywilny oraz niektórych innych ustaw)
[Gutachten II vom 14. Dezember 2010 betr. den Gesetzentwurf Drucksache 2116 (Gesetzentwurf
des Senats zur Änderung des Zivilgesetzbuches und einiger anderer Gesetze)],
http://orka.sejm.gov.pl/rexdomk6.nsf/Opdodr?OpenPage\&nr=2116 [letzter Abruf: 21.10.2015]. 
als beim einfachen Vermächtnis könnte er den Zustand der Unsicherheit auch nicht abkürzen ${ }^{21}$.

Eine solche Situation kann, zumindest mittelbar, die Interessen möglicher Nachlassgläubiger beeinträchtigen. Im polnischen Erbrecht richten sich ihre Ansprüche zwar gegen den Erben und nicht gegen den (einfachen) Vermächtnisnehmer; die Zusammensetzung der Erbmasse kann ihnen aber keineswegs gleichgültig sein.

Alle diese Erwägungen lassen darauf schließen, dass das Fehlen einer Regelung des Alternativvermächtnisses im Zivilgesetzbuch nicht das Ergebnis eines gesetzgeberischen Versehens ist, sondern das Resultat einer bewussten Entscheidung. Hinzu kommt, dass es dieses Institut - darauf weist zu Recht auch der Oberste Gerichtshof hin - in dem Erbrecht von 1946 gab $^{22}$. Dass es nicht in das geltende Recht übernommen wurde, ist also sicher nicht zufällig.

\section{DAS WAHLVERMÄCHTNIS SOWIE BESONDERE ARTEN DES VERMÄCHTNISSES IM DEUTSCHEN RECHT}

Eine Reihe ausländischer Rechtsordnungen kennt das Alternativvermächtnis, u.a. das deutsche Recht. Sedes materiae ist § 2154 BGB. Danach kann der Erblasser ein Vermächtnis in der Art gestalten, dass der Vermächtnisnehmer von mehreren Gegenständen lediglich den einen oder anderen erhalten soll23 (Wahlvermächtnis). Der deutsche Gesetzgeber hat auch eine recht detaillierte Regelung für die Wahl des alternativ festgelegten Vermächtnisgegenstandes getroffen. Das betrifft sowohl das berechtigte Subjekt als auch die Zeit für die Wahl. Im Zweifel ist der Erbe zur Wahl berechtigt. Der Erblasser kann die Auswahl aber auch einer beliebigen dritten Person überlassen.

\footnotetext{
21 U.a. aus diesem Grund regeln die Gesetze, welche expressis verbis das Alternativvermächtnis zulassen, zugleich ziemlich detailliert die mit der Durchführung der Wahl (einschließlich des Zeitpunkts für die Wahl sowie des Übergangs des Wahlrechts auf andere Subjekte) verbundenen Fragen.

22 Dekret über das Erbrecht vom 8.10.1946, Dziennik Ustaw [Gesetzblatt] Nr. 60, Pos. 328.

$23 \S 2154$ Abs. 1 BGB: Der Erblasser kann ein Vermächtnis in der Art anordnen, dass der Bedachte von mehreren Gegenständen nur den einen oder den anderen erhalten soll.
} 
Kann der Berechtigte die Wahl nicht treffen, dann geht das Wahlrecht auf den mit dem Vermächtnis Beschwerten über. Nach allgemeiner Meinung kann ein Gericht (auf Antrag des Vermächtnisnehmers oder des mit dem Vermächtnis Beschwerten) eine Frist für die Durchführung der Wahl festsetzen ${ }^{24}$.

Das Vorhandensein einer ausdrücklichen Regelung des Wahlvermächtnisses im deutschen Recht stellt einerseits eine Bestätigung der Nützlichkeit einer solchen Rechtsfigur dar, zum anderen zeigt es bei der bekannten, weitreichenden Ähnlichkeit der Regelungen des deutschen und des polnischen Rechts in diesem Bereich die Notwendigkeit einer ausdrücklichen Regelung unter bestimmten gesellschaftlichwirtschaftlichen Verhältnissen. Auch nach deutschem Recht muss ein Testament persönlich errichtet werden (§§ 2064 und 2065 BGB). Eine Ausnahme von diesem Grundsatz bedurfte daher einer lex specialis. Eine ähnliche „ausnahmsweise“ Regelung fehlt im polnischen Recht.

Das deutsche Recht kennt übrigens noch andere Arten des Vermächtnisses, die es im polnischen Erbrecht nicht gibt: das Gattungsvermächtnis (§ 2155 BGB), in welchem der Erblasser den Leistungsgegenstand nicht detailliert bestimmt, in dem er sich vielmehr darauf beschränkt, die Gattungseigenschaften einer vermachten Sache aufzuzeigen; das Zweckvermächtnis (§ 2156 BGB), bei dem der Testator lediglich den (üblicherweise wohltätigen) Zweck und evtl. den Begünstigten benennt und es dem billigen Ermessen des Erben überlässt, den Leistungsgegenstand selbst zu bestimmen; das Vermächtnis mit wahlweise Bedachten ( $\$ 2152$ BGB), das dem Erben die Wahl zwischen mehreren Begünstigten überlässt, sowie das aus Sicht des polnischen Erbrechts keine größeren Zweifel hervorrufende Gemeinschaftliche Vermächtnis (§ 2157 BGB), welches mehreren Vermächtnisnehmern eine gleiche oder unterschiedliche Berechtigung an einem Gegenstand zuerkennt.

Der Reichtum deutscher Regelungen wirft die (den Rahmen dieses Aufsatzes überschreitende) Frage auf, ob das polnische Erbrecht nicht

24 W. Edenhofer, [in:] O. Palandt, Bürgerliches Gesetzbuch, München: C.H. Beck 2014, § 2154, Rz. 2. 
auch in diesem noch vom Gesetzgeber der Volksrepublik Polen allzu eng geschneiderten Bereich eine Ergänzung braucht.

\section{SCHLUSSFOLGERUNGEN}

Die Anerkennung der Zulässigkeit des sog. Alternativvermächtnisses bedeutet bei dem in Polen geltenden Rechtszustand eine Verletzung des Grundsatzes, dass ein Testament (und ein sein integrales Element darstellendes Vermächtnis) persönlich zu errichten ist, und ist deshalb de lege lata ausgeschlossen.

Das Fehlen einer Regelung des Alternativvermächtnisses in den Vorschriften des polnischen Erbrechts ist keine zufällige Gesetzeslücke, die man schließen oder im Wege einer freundlichen oder "liberalen“ Auslegung modifizieren kann, wie sie vom polnischen Obersten Gerichtshof in der Entscheidung vom 16. Oktober 2014 vorgenommen worden ist. Bei einer solchen Interpretation käme es zur Kollision mit einer Regel, die ihre tief reichende Begründung hat, nämlich dem Grundsatz einer persönlichen Errichtung des Testaments. Ein - auch nur partieller - Verzicht auf diesen Grundsatz würde - wie im deutschen Recht - eine ausdrückliche gesetzliche Regelung verlangen.

De lege ferenda könnte man überlegen, eine ausdrückliche Regelung in das polnische Recht einzuführen, die ein Alternativvermächtnis zulässt. Ein gesellschaftlicher Bedarf an einer solchen Rechtskonstruktion ist nach meiner Überzeugung aber eher klein, der Preis, den man dafür zu zahlen hätte, beachtlich: Die Unsicherheit etwa für die Erben, die alle Gegenstände als mögliche Objekte des Vermächtnisses betrachten müssten, nähme $\mathrm{zu}$, die Situation von Nachlassgläubigern würde sich möglicherweise verschlechtern. Daher sollte man auch de lege ferenda eher vorsichtig sein. Nach dem Muster des deutschen Rechts und anderer ausländischer Gesetze müsste eine eventuelle Regelung recht detailliert sein, um Unsicherheiten $\mathrm{zu}$ minimalisieren. Zumindest müsste man den Zeitpunkt der Wahl und insbesondere die zur Wahl berechtigten Subjekte bestimmen. 